\title{
Single-cell dissection of a rare human prostate basal cell carcinoma
}

Summary heading : Single-cell analysis of prostate basal cell carcinoma

\section{Abstract}

As a rare subtype of prostate carcinoma, basal cell carcinoma (BCC) has not been studied extensively and thus lacks systematic molecular characterization. Here we applied single-cell genomic amplification and RNA-Seq to a specimen of human prostate $\mathrm{BCC}\left(\mathrm{CK} 34 \beta \mathrm{E} 12^{+} / \mathrm{P}^{+} / \mathrm{PAP}^{-} / \mathrm{PSA}^{-}\right)$. The mutational landscape was obtained via whole exome sequencing of the amplification mixture of 49 single cells, and the 5 putative driver genes mutated are CASC5, NUTM1, PTPRC, KMT2C and TBX3. The top 3 nucleotide substitutions are $\mathrm{C}>\mathrm{T}, \mathrm{T}>\mathrm{C}$ and $\mathrm{C}>\mathrm{A}$, similar to common prostate cancer. The distribution of the variant allele frequency values indicated these single cells are from the same tumor clone. The transcriptomes of 69 single cells were obtained, and they were clustered into tumor, stromal and immune cells based on their global transcriptomic profiles. The tumor cells specifically express basal cell markers like KRT5, KRT14 and KRT23, and epithelial markers EPCAM, CDH1 and CD24. The transcription factor (TF) co-variance network analysis showed that the BCC tumor cells have distinct regulatory networks. By comparison with current prostate cancer datasets, we found that some of the bulk samples exhibit basal-cell signatures. Interestingly, at single-cell resolution the gene expression patterns of prostate $\mathrm{BCC}$ tumor cells show uniqueness compared with that of common prostate cancer-derived circulating tumor cells. This study, for the first time, discloses the comprehensive mutational and transcriptomic landscapes of prostate $\mathrm{BCC}$, which lays a foundation 
for the understanding of its tumorigenesis mechanism and provides new insights into prostate cancers in general.

Xianbin $\mathrm{Su}^{1,2, \#}$, Qi Long ${ }^{3, \#}$, Juanjie $\mathrm{Bo}^{1, \#}, \mathrm{Yi} \mathrm{Shi}^{2, \#}$, Li-Nan $\mathrm{Zhao}^{2}$, Yingxin $\mathrm{Lin}^{4}$, Qing Luo ${ }^{2}$, Shila Ghazanfar ${ }^{4,5}$, Chao Zhang ${ }^{6}$, Qiang Liu ${ }^{7}$, Lan Wang ${ }^{2}$, Kun-Yan $\mathrm{He}^{2}$, Jian $\mathrm{He}^{2}$, Xiao-Fang Cui ${ }^{2}$, Jean Y. H. Yang ${ }^{4}$, Ze-Guang Han ${ }^{2, *}$, Jian-Jun Sha, ${ }^{1, *}$, Guoliang Yang, ${ }^{1, *}$

${ }^{1}$ Department of Urology and Key Laboratory of Systems Biomedicine (Ministry of Education), Renji Hospital, School of Medicine, Shanghai Jiao Tong University, Shanghai, China

${ }^{2}$ Key Laboratory of Systems Biomedicine (Ministry of Education) and Collaborative Innovation Center of Systems Biomedicine, Shanghai Center for Systems Biomedicine, Shanghai Jiao Tong University, Shanghai, China

${ }^{3}$ Key Laboratory for Regenerative Medicine (Ministry of Education), School of Biomedical Sciences, Faculty of Medicine, The Chinese University of Hong Kong, Hong Kong SAR, China

${ }^{4}$ Charles Perkins Center and School of Mathematics and Statistics, The University of Sydney, Sydney, Australia

${ }^{5}$ Cancer Research UK Cambridge Institute, University of Cambridge, Li Ka Shing Centre, Robinson Way, Cambridge CB2 0RE, United Kingdom

${ }^{6}$ Department of Urology, Shanghai Changhai Hospital, Second Military Medical 
University, Shanghai, China

${ }^{7}$ Department of Pathology, Renji Hospital, School of Medicine, Shanghai Jiao Tong University, Shanghai, China

\#These authors contributed equally.

*To whom correspondence should be addressed:

Guoliang Yang, Department of Urology, Renji Hospital, School of Medicine, Shanghai Jiao Tong University, Shanghai 200127, China. Tel: +86-21-6373 0455; Fax: +86-21-6373 0455; Email: yg10511@126.com

Jian-Jun Sha, Department of Urology, Renji Hospital, School of Medicine, Shanghai Jiao Tong University, Shanghai 200127, China. Tel: +86-21-6373 0455; Fax: +86-21-6373 0455; Email: shanjianjunrj@ 126.com

Ze-Guang Han, Key Laboratory of Systems Biomedicine (Ministry of Education), Shanghai Center of Systems Biomedicine, Shanghai Jiao Tong University, Shanghai 200240, China. Tel: +86-21-3420 7304; Fax: +86-21-3420 6059; Email: hanzg@sjtu.edu.cn 


\title{
Running title
}

Single-cell analysis of prostate basal cell carcinoma

\begin{abstract}
Abbreviations
BCC, basal cell carcinoma; CTCs, circulating tumor cells; MDA, multiple displacement amplification; PAP, prostatic acid phosphatase; PSA, prostate-specific antigen; TF, transcription factor; VAF, variant allele frequency.
\end{abstract}

\section{Keywords}

prostate, basal cell carcinoma, single-cell sequencing, gene expression, transcription factor, mutation

\section{Conflict of Interest}

The authors declare that they have no conflicts of interest with the contents of this article. 


\section{Introduction}

Prostate cancer is a heterogeneous disease with complex subtypes and different cell origins [1-3], and basal cell carcinoma (BCC) is an extremely rare histological subtype comprising $<0.01 \%$ of prostate cancer [4]. Despite being largely regarded as pursuing an indolent clinical course, aggressive behaviors like recurrence or metastasis have been observed and deaths also reported, highlighting the complex and yet poorly understood mechanism [5-9]. There have been extensive reports on the genomic features of common prostate cancer [10-17], and the molecular features of normal human prostate basal cells, prostate basal cell hyperplasia, and basal populations from human prostate cancer have also been reported [18-20], but the molecular profile of prostate BCC is still lacking. The mutational and transcriptomic features of this tumor sub-type will thus be valuable for understanding of its tumorigenesis mechanism and development of future clinical treatments.

Single-cell sequencing has evolved to be a powerful tool that provides unprecedented resolution of clinical specimens especially with limited amounts, and marker-free decomposition of the constituent cell types based on single-cell transcriptional profiles allows precise dissection of complex systems such as various tissues or tumors [21-23]. Here we applied single-cell genomic amplification to a human specimen of prostate BCC and used the mixture of 49 single cells to provide mutational landscape of prostate $\mathrm{BCC}$ via whole exome sequencing. We then used single-cell RNA-Seq to obtain the transcriptomes of 69 cells from the same specimen. Three types of cells, namely tumor, stromal and immune cells, were identified with 
their molecular features revealed at single-cell resolution, providing a useful resource for not only this prostate cancer subtype but prostate cancer in general.

\section{Results}

\section{Histological and immunohistochemical features of human prostate BCC}

The tumor exhibited a widespread infiltrative growth pattern under microscopic examination, and individual cells had large pleomorphic nuclei and scant cytoplasm

(Fig. 1). The tumor was negative for two known prostatic markers, prostatic acid phosphatase (PAP) and prostate-specific antigen (PSA), but strongly expressed CK34ßE12 and P63, suggestive of basal cell origin (Fig. 1). Based on the cellular morphology and immunohistochemical staining, we diagnosed it as prostate BCC. The patient with BCC was resistant to chemotherapy but sensitive to radiotherapy, and followed-up for 32 months without recurrence or metastasis (Supporting Information, Fig. S1).

\section{Whole exome sequencing reveals the mutational landscape of prostate BCC}

As genetic variations are generally believed to be the causes of tumors, here we tried to reveal the mutational features of prostate BCC. Due to the limited amount of tumor specimens collected, after dissociation of the specimens into single-cell suspension, we first conducted single-cell whole genome multiple displacement amplification (MDA) on a microfluidic-chip. We then used the mixture of amplified products from 49 single cells for whole exome sequencing (Fig. 2A). A total of 91 functional exonic mutations were obtained, and 5 of the mutated genes, CASC5, NUTM1, PTPRC, 
KMT2C and TBX3 are putative driver genes catalogued in the COSMIC Cancer Gene Census (Supporting Information, Fig. S2) [24]. Mutations in DNA repair genes are related to poor outcomes of prostate cancers $[12,25]$, but few are mutated in this prostate BCC sample such as $B R C A 2$, consistent with good survival of this patient.

The top 3 nucleotide substitutions are $\mathrm{C}>\mathrm{T}, \mathrm{T}>\mathrm{C}$ and $\mathrm{C}>\mathrm{A}$, which are exactly the same top 3 substitutions in recently reported localized, non-indolent prostate tumors (Fig. 2B) [13]. This suggests the etiology of prostate BCC is likely the same as common prostate tumors. The major composite COSMIC signatures are Signature 4, 5, 6 and 12 (Fig. 2C). While the etiologies of Signature 5 and 12 are still unknown, Signature 4 and 6 are associated with smoking and defective DNA mismatch repair, respectively [26].

By checking the distribution of the variant allele frequency (VAF) of the exonic mutations of prostate $\mathrm{BCC}$, it is clear that most of the variations have VAF values of around 0.5 , thus supporting that most of the single cells share a similar mutational background (Fig. 2D). The VAF values of the 5 driver mutations are also approximately 0.5 (Fig. 2E), indicating these single cells are probably originated from the same ancestor and form the major tumor clone. However, there are still some variations with lower VAF values which suggest sub-clonal mutations may have existed in some cells that are acquired during later diversified evolution of the tumor.

To understand whether the prostate BCC is showing similarities to other reported prostate cancer cases, we checked the mutational frequency of the prostate BCC mutated genes in prostate cancer samples collected in cBioPortal [27]. For the 88 
genes enquired, only 10 genes are not mutated, and 27 genes are mutated in more than $0.5 \%$ of the $\sim 4400$ prostate cancer samples (Fig. $2 \mathrm{~F}$ ). Interestingly, 3 of the driver genes from this study, $K M T 2 C$, PTPRC, and TBX3 are also among the top mutated genes, especially $K M T 2 C$ with mutation rate of $\sim 6 \%$. GSEA/MSigDB enrichment analysis [28] of the mutated genes shows that the top enriched terms are related to cell cycle, cytoskeleton and others (Fig. 2G), consistent with its basal cell origin.

\section{Single-cell RNA-Seq reveals the transcriptomic features of prostate BCC}

To understand the transcriptional profiles of prostate $\mathrm{BCC}$, we then randomly selected 69 single cells for single-cell RNA-Seq (Fig. 3A). With a median unique mapping rate of $58.4 \%$ and 1.08 million mapped reads, we obtained a median of $\sim 2800$ genes per cell (Supporting Information, Fig. S3A). We used ERCC spike-ins as positive controls, and the high correlation efficiency between single-cells based on the 92 spike-ins confirmed the high quality of the data (Supporting Information, Fig. S3B-D). After filtering 5 outliers, the remaining 64 cells were classified into three clusters based on their global transcriptomic profiles (Fig. 3B-C). GSEA/MSigDB enrichment analysis identified them as tumor cells (51 cells), stromal cells ( 7 cells) and immune cells (6 cells) (Fig. 3D).

The three clusters of cells can also be clearly separated in tSNE plot based on the top genes expressed in each cluster (Fig. 4A). The tumor cells specifically expressed genes encoding cytokeratins including KRT5, KRT14 and KRT23, which are reliable basal cell markers (Fig. 4B). Interestingly, epithelial markers EPCAM, CDH1 and 
CD24 are also highly expressed in tumor cells. Stromal cells specifically express COL1A2, THY1 and BGN, and immune cells specifically express monocytic marker CD14, CXCL8 and myeloid cell nuclear differentiation antigen MNDA (Fig. 4B), suggestive of possible innate immune cell infiltration. Immunohistochemical staining confirmed the expression of CK14 (KRT14) at protein level, further proving the reliability of the sequencing data (Fig. 4C).

Transcription factors (TFs) covariance network analysis showed that the three clusters demonstrate different transcriptional regulatory status (Fig. 4D). Significantly, MYC, ARIDIA, TBX3 and SOX11 are highly expressed in tumor cells, where, besides the well-known MYC that is recently reported to be over-expressed in human prostatic basal cells [19], the oncogenic role of ARIDIA, a key component of chromatin remodeling complex SWI/SNF, has been recently demonstrated in liver cancer [29], the development-related gene SOX11 was reported to be elevated in basal-like breast cancer [30], and $T B X 3$ plays important roles in development, differentiation and tumorigenesis [31]. The TFs co-variance network provides clues to future dissection of the regulatory mechanism of BCC.

\section{Single-cell analysis of prostate BCC provides new insights to prostate cancer}

We then checked the expression of prostate BCC tumor cell specific genes in previously reported luminal and basal cells sorted from normal and cancerous prostates by flow cytometry [18]. The prostate basal cells exhibit higher expression levels of KRT5, KRT14 and KRT23 compared with luminal cells, similarly as our 
prostate BCC tumor cells, further confirming their basal cell origin (Fig. 5A). Interestingly, the genes highly expressed in prostate BCC tumor cells fall into two groups based on their expression correlation patterns, with one group including KRT5, KRT14, KRT23, DSC3, FGFR2, etc., while the other group including EPCAM, CDH1, $C D 24$, etc. (Fig. 5A). It seems the former group includes the basal cell markers while the latter group includes genes expressed in common prostate epithelial cells.

We further checked the expression patterns of BCC tumor cell specific genes in other bulk samples of human prostate cancers $[10,11,32]$. It is interesting to identify a small group of samples exhibiting basal cell gene expression features in two separate prostate cancer datasets (shaded branches in the dendrograms of Fig. 5B and Supporting Information, Fig. S4A). Likely explanations are that these tumor samples were derived from BCC patients or the samples contained basal cells. For TCGA PRAD dataset, the expressions of basal markers are detected in most samples, possibility due to the high number of genes detected by deep sequencing (Supporting Information, Fig. S4B). The split of the genes highly expressed in BCC tumor cells into 2 groups is further confirmed by these three datasets, with $K R T 5, K R T 14, K R T 23$ clustering together with $D S C 3, F G F R 2$ and also possibly ACTG2. Our single-cell analysis of prostate BCC reveals the existence of basal cell features in current bulk samples of prostate cancer.

We then compared our results with one single-cell resolution dataset of prostate cancer [33]. Compared with circulating tumor cells (CTCs) from prostate cancer patients, the prostate $\mathrm{BCC}$ tumor cells exhibit distinct gene expression features with 
high levels of IGF2, KRT5, KRT14, KRT23, AQP1, MIA, FGFR2 and ACTG2, although they all expressed EPCAM, CDH1 and CD24 (Fig. 5C). The results demonstrated the unique gene expression patterns of the prostate BCC tumor cells, which exhibit the molecular features of basal cells with activated genes like IGF2 and FGFR2, consistent with report on the regulation of pluripotency by IGF and FGF pathways [34]. Another interesting finding is that the BCC immune cells clustered together with some single cells annotated as CTC-candidate, which were likely to be contaminating leukocytes to the CTCs (Fig. 5C). The single-cell level data also support the above mentioned split of the genes highly expressed in prostate BCC tumor cells, with one group representing genuine BCC markers while the other genes commonly expressed in prostate cancer.

\section{Detection of exonic mutations in single-cell RNA-Seq reads}

As our mutational data and single-cell RNA-Seq data were generated from the same prostate BCC specimen, we checked whether the exonic mutations could be detected in RNA-Seq reads. Interestingly, we indeed found mutations in RNA-Seq reads from some single cells for $G A B P B 2, A S T E 1, C O P S 3$ and BEX2 (Supporting Information, Fig. S5). The variations are only detected in some of the single cells, which are likely because the mutated sites are not always covered by the relatively shallow single-cell RNA-Seq. Another phenomenon is that single cells in RNA-Seq analysis only contained either reference or altered variant, which is probably caused by allelic 
specific expression or allele dropout in RNA-Seq. The consistency of scRNA-Seq data and exome-seq data further suggested the reliability of our prostate BCC data.

\section{Discussion}

As a rare malignancy, there has been little research into the molecular characteristics of prostate $\mathrm{BCC}$ and no consensus on its treatment. Mostly, such data exist as sporadic reports on immunohistochemical expression in a small number of cases $[8$, 35-37]. This study represents the first genomic and transcriptomic profiling of this prostate cancer subtype, and the single-cell resolution transcriptomic data is especially valuable for understanding of not only this subtype but prostate cancer in general.

The molecular features of normal human prostate basal cells [19], prostate basal cell hyperplasia [20], and human prostate cancer derived basal populations [18] have been reported recently, and the single-cell transcriptomic profiles of prostate BCC is a good complement to these data for comparison studies and better understanding of prostate cancer cell origin. Most of the current genomics data for prostate cancer are generated using bulk samples $[10,11,13]$, and the single-cell transcriptional profiles of prostate BCC provide an opportunity for deeper utilization of these data. For example, analysis using genes highly expressed in prostate BCC reveals the existence of basal cell features in some bulk samples of prostate cancer, which is consistent with recent classification of prostate cancer samples into basal-like and luminal-like subtypes using the PAM50 classifier [1, 38]. The single-cell resolution profiles of constitutional cell types from prostate BCC provide further advantage for future 
de-convolution of bulk samples [39], which will maximize the values of current genomic data of prostate cancer.

The prostate BCC patient is still surviving now, indicating this case is an indolent malignancy. Our genomics and transcriptomic analysis of this chemotherapy-resistant but radiotherapy-sensitive prostate $\mathrm{BCC}$ provides a useful resource for future dissection of the molecular mechanisms related to the radiotherapy responsiveness and treatment guidance. Mutations that disrupt the function of DNA damage repair genes have been shown to be associated with the aggressive clinical behavior of localized prostate cancer and with cancer-specific mortality [12, 40-42], and it has also been reported that men with metastatic prostate cancer and DNA-repair gene mutations have sustained responses to poly-ADP ribose polymerase (PARP) inhibitors and platinum-based chemotherapy [43, 44]. This study showed that the prostate BCC patient with few DNA-repair gene mutations was resistant to chemotherapy, suggesting that we may directly adopt radiotherapy rather than chemotherapy for such conditions. The results indicated that precision treatment of BCC could be more than just a histopathological triviality but be based on association of tumor phenotypes with molecular features.

In summary, single-cell analysis revealed the genomic and transcriptomic landscapes of the rare prostate BCC for the first time. This provides clues for elucidation of the tumorigenesis mechanism, further discovery of biomarkers and therapeutic targets, and better understanding of not only prostate $\mathrm{BCC}$ but prostate cancer in general. 


\section{Methods}

\section{Clinical specimens}

This study was approved by the Ethnical Review Board of Renji Hospital, School of Medicine, Shanghai Jiao Tong University. Specimens were obtained by prostatic needle biopsy from a 55-year-old man with pelvic pain and irritative urinary symptoms. The specimens underwent pathological evaluation. After diagnosis as prostate $\mathrm{BCC}$, the patient was treated with chemotherapy and radiotherapy and then followed-up for 32 months without evidence of local recurrence or distant metastases (Supporting Information, Fig. S1). The specimens were mechanically dissociated and digested with collagenase IV and DNase I, and the single-cell suspensions were used for both single-cell genome amplification and single-cell RNA-Seq.

\section{Immunohistochemical staining}

Immunohistochemical staining was done as previously described [45], and we used the following primary antibodies: P63 (abcam, ab124762), CK34ßE12 (Dako, M0630), CK14 (abcam, ab7800), PAP (Dako, M0792), PSA (Dako, A056201). We used biotinylated universal link antibody as the secondary antibody.

\section{Single-cell genome amplification and whole exome sequencing}

Single-cell capture, lysis, reverse-transcription, and whole genome MDA were done in a microfluidic-based C1 DNA-Seq IFC (10 17 $\mu \mathrm{m}$, Fluidigm) according to its 
protocol using illustra GenomiPhi V2 DNA Amplification Kit (GE Healthcare, 25660031). The amplified products from 49 single cells were mixed for exonic region capture and library preparation using Agilent SureSelect Human All Exon v7 Kit (Agilent, 5191-4005). The library was sequenced using illumina HiSeq platform with $2 \times 150$ bp sequencing mode. We used GATK [46] for variant calling and ANNOVAR [47] for functional annotation of the mutations. As there is no para-tumor tissue for this patient, we filtered SNPs using dbSNP141 [48] and 1,000 Genomes Project (v3) database [49]. We used MutationalPatterns [50] to decipher the mutational signature composition. For genes mutated in this prostate BCC sample, we checked their mutational frequencies in prostate cancer samples collected in cBioPortal [27]. GSEA/MSigDB enrichment analysis was also conducted for the genes mutated in this prostate $\mathrm{BCC}$.

\section{Single-cell RNA-Seq and data analysis}

Single-cell RNA-Seq experiment and analysis were conducted as previously described [45]. Single-cell capture, lysis, reverse-transcription, and cDNA amplification were done in a microfluidic-based C1 RNA-Seq IFC (10 17 $\mu \mathrm{m}$, Fluidigm). After library preparation with Nextera XT Kit and Index Kit (illumina), the single-cell libraries were pooled and sequenced using NextSeq 500 (illumina) with 2 $\times 76$ bp sequencing mode. After generation of FPKM data, SC3 [51] was used for identification of cell outliers and single-cell clustering. The genes differently expressed between clusters were then identified via ANOVA analysis $(p<0.05)$ using 
SINGuLAR ${ }^{\mathrm{TM}}$ (Fluidigm). GSEA/MSigDB enrichment analysis was conducted for the genes specifically expressed in each cluster to facilitate cell type identification. Transcription factors (TFs) covariance network analysis was conducted to reveal the relationship of the TFs specifically expressed in each cell cluster as previously described [45, 52]. Gene expression files were retrieved from five other prostate cancer studies $[10,11,18,32,33]$ for comparison studies.

\section{Acknowledgement}

This work is supported in part by the National Natural Science Foundation of China (81802806, 81472621, 81402329 and 81902561), National Program on Key Research Project of China (2016YFC0902701, Precision Medicine), Shanghai Health Bureau (20164Y0124), Medical and Engineering Crossover Fund of SJTU (YG2016QN71, YG2017MS67), University of Sydney - Shanghai Jiao Tong University Joint Research Alliance (USYD-SJTU JRA) grant, funding from Key Laboratory of Systems Biomedicine (Ministry of Education) (KLSB2017QN-03), and Incubating Program for Clinical Research and Innovation of Renji Hospital SJTU School of Medicine (PYII-17-010).

\section{Authors' contributions}

XS and GY designed the study. XS, JB, LZ, CZ, QL3, LW, KH, JH, XC, WX and GY carried out experiments. XS, QL1, YS, YL, QL2, SG and JY analyzed data. XS, GY and ZH drafted the manuscript. GY, JS and ZH supervised the study. All authors read and approved final version of the manuscript. 


\section{References}

1. Zhao SG, Chang SL, Erho N, Yu M, Lehrer J, Alshalalfa M, Speers C, Cooperberg MR, Kim W, Ryan CJ, et al. (2017) Associations of Luminal and Basal Subtyping of Prostate Cancer With Prognosis and Response to Androgen Deprivation Therapy. JAMA Oncol 3, 1663-1672.

2. Goldstein AS, Huang J, Guo C, Garraway IP \& Witte ON (2010) Identification of a cell of origin for human prostate cancer. Science 329, 568-571.

3. Choi N, Zhang B, Zhang L, Ittmann M \& Xin L (2012) Adult murine prostate basal and luminal cells are self-sustained lineages that can both serve as targets for prostate cancer initiation. Cancer Cell 21, 253-265.

4. Tannenbaum M (1975) Adenoid cystic or "salivary gland" carcinomas of prostate. Urology 6, 238-239.

5. Halat SK \& MacLennan GT (2008) Adenoid cystic/basal cell carcinoma of the prostate. J Urol 179, 1576.

6. Komura K, Inamoto T, Tsuji M, Ibuki N, Koyama K, Ubai T, Azuma H \& Katsuoka Y (2010) Basal cell carcinoma of the prostate: unusual subtype of prostatic carcinoma. Int J Clin Oncol 15, 594-600.

7. Chang K, Dai B, Kong Y, Qu Y, Wu J, Ye D, Yao X, Zhang S, Zhang H, Zhu Y, et al. (2013) Basal cell carcinoma of the prostate: clinicopathologic analysis of three cases and a review of the literature. World J Surg Oncol 11, 193.

8. Ali TZ \& Epstein JI (2007) Basal cell carcinoma of the prostate: a clinicopathologic study of 29 cases. Am J Surg Pathol 31, 697-705.

9. Ayyathurai R, Civantos F, Soloway MS \& Manoharan M (2007) Basal cell carcinoma of the prostate: current concepts. BJU Int 99, 1345-1349.

10. The Cancer Genome Atlas Research Network (2015) The Molecular Taxonomy 
of Primary Prostate Cancer. Cell 163, 1011-1025.

11. Robinson D, Van Allen EM, Wu YM, Schultz N, Lonigro RJ, Mosquera JM, Montgomery B, Taplin ME, Pritchard CC, Attard G, et al. (2015) Integrative clinical genomics of advanced prostate cancer. Cell 161, 1215-1228.

12. Pritchard CC, Mateo J, Walsh MF, De Sarkar N, Abida W, Beltran H, Garofalo A, Gulati R, Carreira S, Eeles R, et al. (2016) Inherited DNA-Repair Gene Mutations in Men with Metastatic Prostate Cancer. N Engl J Med 375, 443-453.

13. Fraser M, Sabelnykova VY, Yamaguchi TN, Heisler LE, Livingstone J, Huang V, Shiah YJ, Yousif F, Lin X, Masella AP, et al. (2017) Genomic hallmarks of localized, non-indolent prostate cancer. Nature 541, 359-364.

14. Grasso CS, Wu YM, Robinson DR, Cao X, Dhanasekaran SM, Khan AP, Quist MJ, Jing X, Lonigro RJ, Brenner JC, et al. (2012) The mutational landscape of lethal castration-resistant prostate cancer. Nature 487, 239-243.

15. Quigley DA, Dang HX, Zhao SG, Lloyd P, Aggarwal R, Alumkal JJ, Foye A, Kothari V, Perry MD, Bailey AM, et al. (2018) Genomic Hallmarks and Structural Variation in Metastatic Prostate Cancer. Cell 174, 758-769.

16. Espiritu SMG, Liu LY, Rubanova Y, Bhandari V, Holgersen EM, Szyca LM, Fox NS, Chua MLK, Yamaguchi TN, Heisler LE, et al. (2018) The Evolutionary Landscape of Localized Prostate Cancers Drives Clinical Aggression. Cell 173, 1003-1013 e1015.

17. Baca SC, Prandi D, Lawrence MS, Mosquera JM, Romanel A, Drier Y, Park K, Kitabayashi N, MacDonald TY, Ghandi M, et al. (2013) Punctuated evolution of prostate cancer genomes. Cell 153, 666-677.

18. Smith BA, Sokolov A, Uzunangelov V, Baertsch R, Newton Y, Graim K, Mathis C, Cheng D, Stuart JM \& Witte ON (2015) A basal stem cell signature identifies 
aggressive prostate cancer phenotypes. Proc Natl Acad Sci U S A 112, E6544-6552.

19. Zhang D, Park D, Zhong Y, Lu Y, Rycaj K, Gong S, Chen X, Liu X, Chao HP, Whitney P, et al. (2016) Stem cell and neurogenic gene-expression profiles link prostate basal cells to aggressive prostate cancer. Nat Commun 7, 10798.

20. Henry G, Malewska A, Mauck R, Gahan J, Hutchinson R, Torrealba J, Francis F, Roehrborn C \& Strand D (2017) Molecular pathogenesis of human prostate basal cell hyperplasia. Prostate 77, 1344-1355.

21. Gao S, Yan L, Wang R, Li J, Yong J, Zhou X, Wei Y, Wu X, Wang X, Fan X, et al. (2018) Tracing the temporal-spatial transcriptome landscapes of the human fetal digestive tract using single-cell RNA-sequencing. Nat Cell Biol 20, 721-734.

22. Han X, Wang R, Zhou Y, Fei L, Sun H, Lai S, Saadatpour A, Zhou Z, Chen H, Ye F, et al. (2018) Mapping the Mouse Cell Atlas by Microwell-Seq. Cell 172, 1091-1107 e1017.

23. Tanay A \& Regev A (2017) Scaling single-cell genomics from phenomenology to mechanism. Nature 541, 331-338.

24. Sondka Z, Bamford S, Cole CG, Ward SA, Dunham I \& Forbes SA (2018) The COSMIC Cancer Gene Census: describing genetic dysfunction across all human cancers. Nat Rev Cancer 18, 696-705.

25. Taylor RA, Fraser M, Rebello RJ, Boutros PC, Murphy DG, Bristow RG \& Risbridger GP (2019) The influence of BRCA2 mutation on localized prostate cancer. Nat Rev Urol 16, 281-290.

26. Alexandrov LB, Nik-Zainal S, Wedge DC, Aparicio SA, Behjati S, Biankin AV, Bignell GR, Bolli N, Borg A, Borresen-Dale AL, et al. (2013) Signatures of mutational processes in human cancer. Nature 500, 415-421.

27. Gao J, Aksoy BA, Dogrusoz U, Dresdner G, Gross B, Sumer SO, Sun Y, 
Jacobsen A, Sinha R, Larsson E, et al. (2013) Integrative analysis of complex cancer genomics and clinical profiles using the cBioPortal. Sci Signal 6, pl1.

28. Subramanian A, Tamayo P, Mootha VK, Mukherjee S, Ebert BL, Gillette MA, Paulovich A, Pomeroy SL, Golub TR, Lander ES, et al. (2005) Gene set enrichment analysis: a knowledge-based approach for interpreting genome-wide expression profiles. Proc Natl Acad Sci U S A 102, 15545-15550.

29. Sun X, Wang SC, Wei Y, Luo X, Jia Y, Li L, Gopal P, Zhu M, Nassour I, Chuang JC, et al. (2017) Arid1a has context-dependent oncogenic and tumor suppressor functions in liver cancer. Cancer Cell 32, 574-589 e576.

30. Shepherd JH, Uray IP, Mazumdar A, Tsimelzon A, Savage M, Hilsenbeck SG \& Brown PH (2016) The SOX11 transcription factor is a critical regulator of basal-like breast cancer growth, invasion, and basal-like gene expression. Oncotarget 7, 13106-13121.

31. Wansleben S, Peres J, Hare S, Goding CR \& Prince S (2014) T-box transcription factors in cancer biology. Biochim Biophys Acta 1846, 380-391.

32. Beltran H, Prandi D, Mosquera JM, Benelli M, Puca L, Cyrta J, Marotz C, Giannopoulou E, Chakravarthi BV, Varambally S, et al. (2016) Divergent clonal evolution of castration-resistant neuroendocrine prostate cancer. Nat Med 22, 298-305.

33. Miyamoto DT, Zheng Y, Wittner BS, Lee RJ, Zhu H, Broderick KT, Desai R, Fox DB, Brannigan BW, Trautwein J, et al. (2015) RNA-Seq of single prostate CTCs implicates noncanonical Wnt signaling in antiandrogen resistance. Science 349, $1351-1356$.

34. Bendall SC, Stewart MH, Menendez P, George D, Vijayaragavan K, Werbowetski-Ogilvie T, Ramos-Mejia V, Rouleau A, Yang J, Bosse M, et al. (2007) IGF and FGF cooperatively establish the regulatory stem cell niche of pluripotent 
human cells in vitro. Nature 448, 1015-1021.

35. Yang XJ, McEntee M \& Epstein JI (1998) Distinction of basaloid carcinoma of the prostate from benign basal cell lesions by using immunohistochemistry for bcl-2 and Ki-67. Hum Pathol 29, 1447-1450.

36. Iczkowski KA, Ferguson KL, Grier DD, Hossain D, Banerjee SS, McNeal JE \& Bostwick DG (2003) Adenoid cystic/basal cell carcinoma of the prostate: clinicopathologic findings in 19 cases. Am J Surg Pathol 27, 1523-1529.

37. Montironi R, Mazzucchelli R, Stramazzotti D, Scarpelli M, Lopez Beltran A \& Bostwick DG (2005) Basal cell hyperplasia and basal cell carcinoma of the prostate: a comprehensive review and discussion of a case with c-erbB-2 expression. J Clin Pathol 58, 290-296.

38. Zhao SG, Chen WS, Das R, Chang SL, Tomlins SA, Chou J, Quigley DA, Dang HX, Barnard TJ, Mahal BA, et al. (2019) Clinical and Genomic Implications of Luminal and Basal Subtypes Across Carcinomas. Clin Cancer Res 25, 2450-2457.

39. Wang X, Park J, Susztak K, Zhang NR \& Li M (2019) Bulk tissue cell type deconvolution with multi-subject single-cell expression reference. Nat Commun 10, 380.

40. Leongamornlert D, Saunders E, Dadaev T, Tymrakiewicz M, Goh C, Jugurnauth-Little S, Kozarewa I, Fenwick K, Assiotis I, Barrowdale D, et al. (2014) Frequent germline deleterious mutations in DNA repair genes in familial prostate cancer cases are associated with advanced disease. Br J Cancer 110, 1663-1672.

41. Castro E, Goh C, Olmos D, Saunders E, Leongamornlert D, Tymrakiewicz M, Mahmud N, Dadaev T, Govindasami K, Guy M, et al. (2013) Germline BRCA mutations are associated with higher risk of nodal involvement, distant metastasis, and poor survival outcomes in prostate cancer. J Clin Oncol 31, 1748-1757. 
42. Gallagher DJ, Gaudet MM, Pal P, Kirchhoff T, Balistreri L, Vora K, Bhatia J, Stadler Z, Fine SW, Reuter V, et al. (2010) Germline BRCA mutations denote a clinicopathologic subset of prostate cancer. Clin Cancer Res 16, 2115-2121.

43. Cheng HH, Pritchard CC, Boyd T, Nelson PS \& Montgomery B (2016) Biallelic Inactivation of BRCA2 in Platinum-sensitive Metastatic Castration-resistant Prostate Cancer. Eur Urol 69, 992-995.

44. Mateo J, Carreira S, Sandhu S, Miranda S, Mossop H, Perez-Lopez R, Nava Rodrigues D, Robinson D, Omlin A, Tunariu N, et al. (2015) DNA-Repair Defects and Olaparib in Metastatic Prostate Cancer. N Engl J Med 373, 1697-1708.

45. Su X, Shi Y, Zou X, Lu Z-N, Xie G, Yang JYH, Wu C-C, Cui X-F, He K-Y, Luo Q, et al. (2017) Single-cell RNA-Seq analysis reveals dynamic trajectories during mouse liver development. BMC Genomics 18, 946.

46. McKenna A, Hanna M, Banks E, Sivachenko A, Cibulskis K, Kernytsky A, Garimella K, Altshuler D, Gabriel S, Daly M, et al. (2010) The Genome Analysis Toolkit: a MapReduce framework for analyzing next-generation DNA sequencing data. Genome Res 20, 1297-1303.

47. Wang K, Li M \& Hakonarson H (2010) ANNOVAR: functional annotation of genetic variants from high-throughput sequencing data. Nucleic Acids Res 38, e164.

48. Sherry ST, Ward MH, Kholodov M, Baker J, Phan L, Smigielski EM \& Sirotkin K (2001) dbSNP: the NCBI database of genetic variation. Nucleic Acids Res 29, 308-311.

49. Auton A, Brooks LD, Durbin RM, Garrison EP, Kang HM, Korbel JO, Marchini JL, McCarthy S, McVean GA \& Abecasis GR (2015) A global reference for human genetic variation. Nature 526, 68-74.

50. Blokzijl F, Janssen R, van Boxtel R \& Cuppen E (2018) MutationalPatterns: 
comprehensive genome-wide analysis of mutational processes. Genome Med 10, 33.

51. Kiselev VY, Kirschner K, Schaub MT, Andrews T, Yiu A, Chandra T, Natarajan KN, Reik W, Barahona M, Green AR, et al. (2017) SC3: consensus clustering of single-cell RNA-seq data. Nat Methods 14, 483-486.

52. Zhang HM, Liu T, Liu CJ, Song S, Zhang X, Liu W, Jia H, Xue Y \& Guo AY (2015) AnimalTFDB 2.0: a resource for expression, prediction and functional study of animal transcription factors. Nucleic Acids Res 43, D76-81. 


\section{Figure Legend}

Fig. 1. Histological and immunohistochemical staining of the prostate BCC. H\&E staining and the expressions of CK34ßE12, P63, PAP and PSA are shown.

Fig. 2. Whole exome sequencing reveals the mutational landscape of prostate BCC.

(A) General description of the exome sequencing of mixtures from genomic amplification products of prostate BCC single cells and numbers of genetic variations.

(B) Mutational spectra of the prostate BCC based on the variants after SNP filter. (C)

Contributions of COSMIC signatures to the prostate $\mathrm{BCC}$ mutational spectra. (D)

Distribution of the variant allele frequency (VAF) of the exonic mutations of prostate

BCC. (E) Summary of the driver (overlapped with The Cancer Gene Census) and putative sub-clonal (VAF lower than 0.35) mutations of prostate BCC. (F) Percent of samples mutated in the cBioPortal prostate cancer datasets with those above $1 \%$ shown for the exonic mutated genes in prostate BCC. The red stars indicate Census driver genes. $(\mathrm{G})$ The items enriched with the exonic mutated genes in prostate BCC via GSEA/MSigDB canonical pathway enrichment analysis.

Fig. 3. Single-cell RNA-Seq reveals the constituent cell types in the prostate BCC. (A) Experimental workflow. (B) Heat-map showing the single-cell gene-expression patterns of the three clusters identified. (C) Heat-map showing the Pearson correlation coefficients between single-cells. (D) The top 10 items enriched with the gene sets specifically expressed in each cell cluster via GSEA/MSigDB canonical pathway 
enrichment analysis.

Fig. 4. Single-cell RNA-Seq reveals the transcriptomic features of prostate BCC. (A) Heat-map showing the expression patterns of top 20 genes specifically and highly expressed in each cluster, and t-SNE plot of the three clusters. (B) The expression of selected markers with the median values and the first and third quartiles shown. (C) Immunohistochemical staining of the specimen with CK14 antibody. (D) TFs covariance networks of the single-cell data. Each node represents a TF, and each edge represents correlation between two TFs. The TFs specifically expressed in each cluster are colored differently.

Fig. 5. Single-cell analysis of prostate BCC provide new insights to prostate cancer. (A) (left) Heat-map showing the clustering of FACS sorted luminal (CD49f Lo) and basal (CD49f Hi) cell populations from human benign and cancerous prostates based on the genes highly expressed in prostate BCC tumor cells; (right) Heat-map showing the Pearson correlation coefficients between the genes. (Data from Smith et al. PNAS 2015; 112: E6544-E6552). (B) Analysis of prostate cancer samples in similar approach as (A). (Data from Robinson et al. Cell 2015; 161: 1215-1228). (C) Comparison of the gene expression profiles of single cells from Cluster 1 3 of prostate BCC with human prostate CTCs (CTC-candidate and CTC-confirmed), bulk primary tumors, single cells from prostate cancer cell lines (Pca cell line) and white blood cells (Data from Miyamoto et al. Science 2015; 349: 1351-1356). 
Fig 1

H\&E

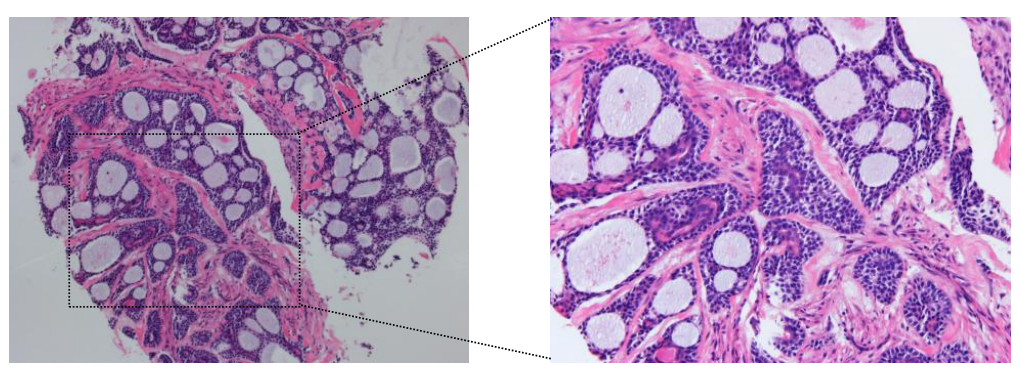

CK34ßE12

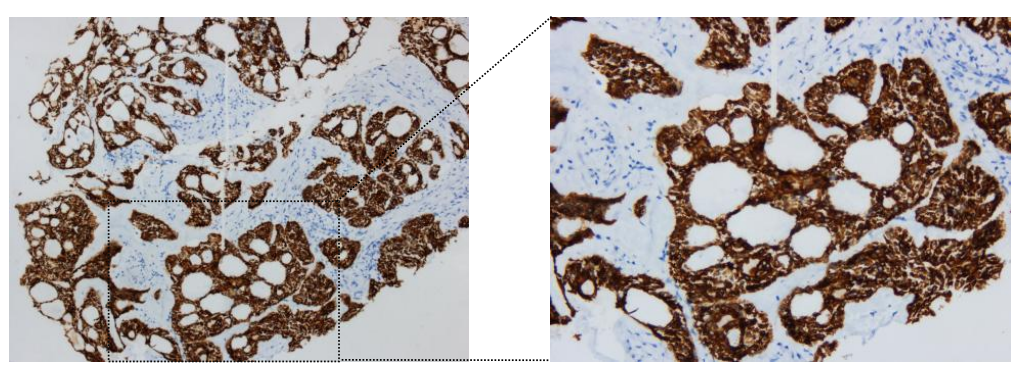

P63
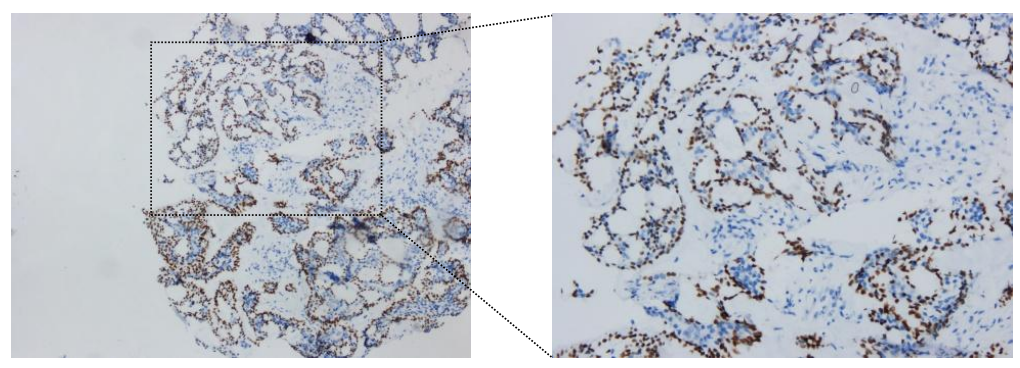

PAP
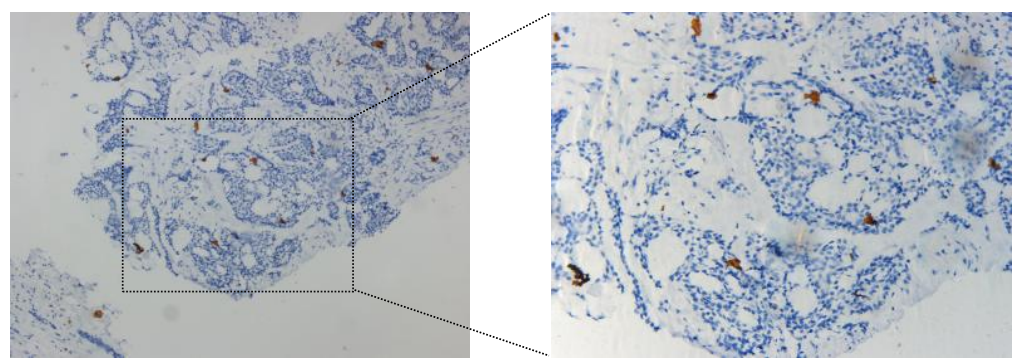

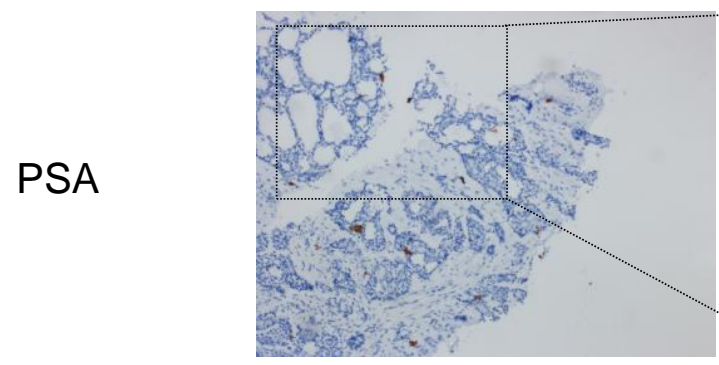

$100 \times$

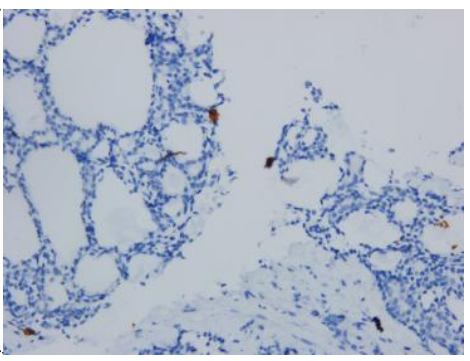

$200 \times$ 
A

Whole genome amplification of BCC single cells $\downarrow$

Whole exome sequencing (Mixture of 49 cells)

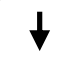

7395 variations after SNP filter

$\downarrow$

91 exonic variations

$\downarrow$

5 genes overlap with Census

D

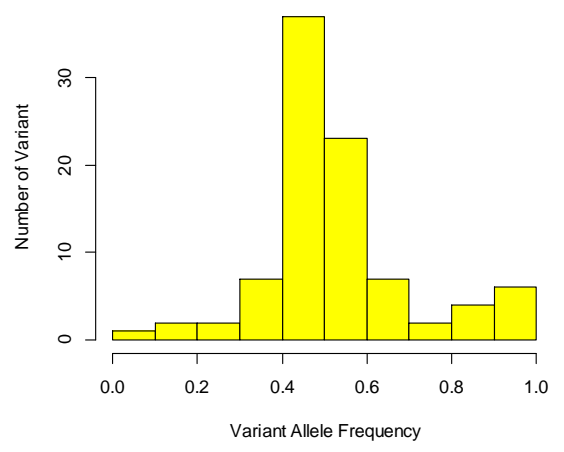

F

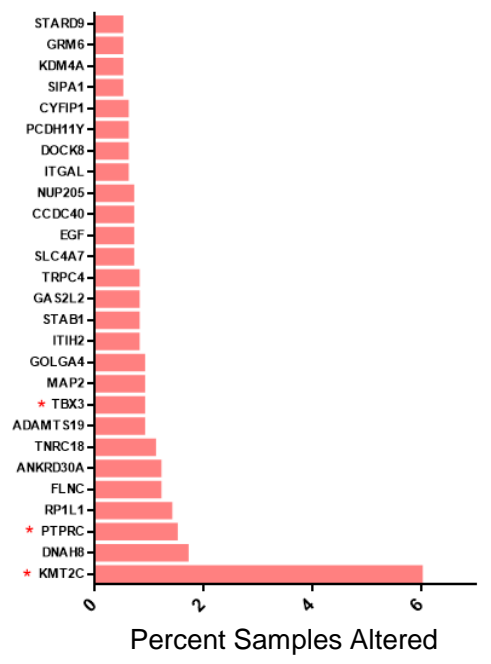

B
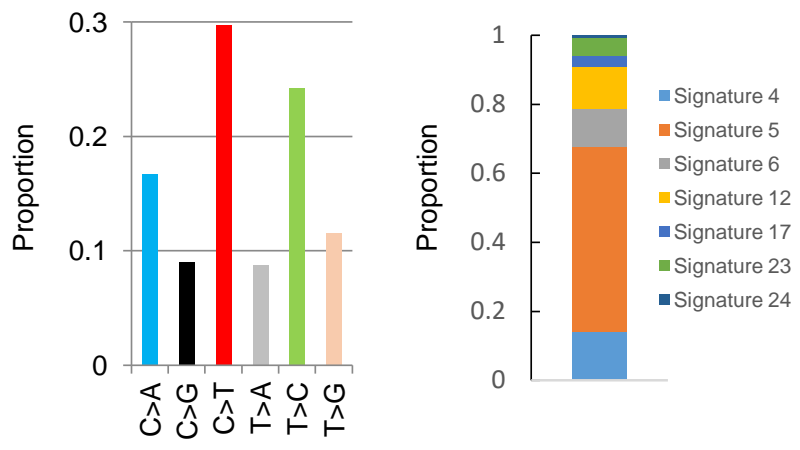

E

\begin{tabular}{|c|c|c|c|c|c|c|c|}
\hline Group & Gene & \#Chr & Position & Ref & Alt & AA change & VAF \\
\hline \multirow{5}{*}{$\begin{array}{l}\text { 올. } \\
\text { Ф }\end{array}$} & CASC5 & 15 & 40903002 & $A$ & $G$ & S86G & 0.68 \\
\hline & NUTM1 & 15 & 34640367 & $\mathrm{G}$ & C & V90L & 0.63 \\
\hline & PTPRC & 1 & 198713275 & $\mathrm{~T}$ & A & $\mathrm{H} 769 \mathrm{Q}$ & 0.59 \\
\hline & КМт2C & 7 & 151845915 & $A$ & $\mathrm{~T}$ & $14366 \mathrm{~N}$ & 0.54 \\
\hline & TBX3 & 12 & 115112422 & C & G & A420P & 0.42 \\
\hline \multirow{9}{*}{ 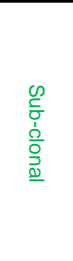 } & KRT18 & 12 & 53343099 & $\mathrm{G}$ & $A$ & V48M & 0.33 \\
\hline & TNRC18 & 7 & 5360200 & $\mathrm{G}$ & A & P2200L & 0.33 \\
\hline & KRT18 & 12 & 53343132 & $\mathrm{G}$ & $\mathrm{T}$ & G59W & 0.33 \\
\hline & SCUBE3 & 6 & 35213742 & C & $\mathrm{T}$ & T874I & 0.30 \\
\hline & KRT18 & 12 & 53343148 & C & $G$ & $\mathrm{~A} 64 \mathrm{G}$ & 0.27 \\
\hline & ART5 & 11 & 3661519 & $\mathrm{~T}$ & $\mathrm{G}$ & $\mathrm{E} 47 \mathrm{~A}$ & 0.25 \\
\hline & MST1L & 1 & 17084321 & A & $\mathrm{G}$ & C566R & 0.17 \\
\hline & CCDC40 & 17 & 78064020 & C & $A$ & T972K & 0.12 \\
\hline & C7orf26 & 7 & 6647724 & $\mathrm{G}$ & C & $\mathrm{A} 428 \mathrm{P}$ & 0.09 \\
\hline
\end{tabular}

G

GO_single_organism_cellular_loca..

GO_cell_cycle_process

kegg_cell_adhesion_molecules_ca.

GO_negative_regulation_of_cataly GO_regulation_of_hydrolase_activity GO_intracellular_signal_transduction GO_regulation_of_microtubule_ba.. GO_cell_cycle GO_microtubule_bundle_formation GO_microtubule_based_process GO_microtubule_cytoskeleton_org. GO_cytoskeleton_organization GO_regulation_of_cell_cycle

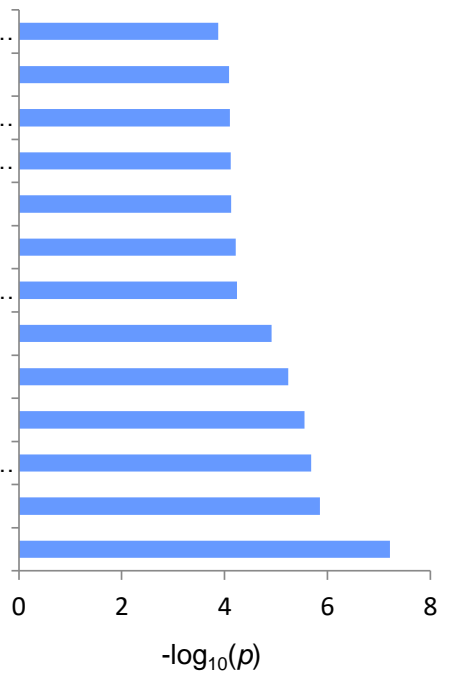




\section{A $\quad$ Fig 3}

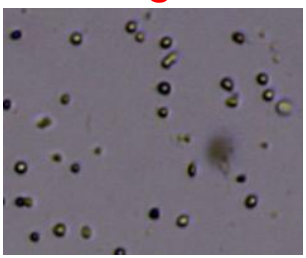

Single cells(SC)

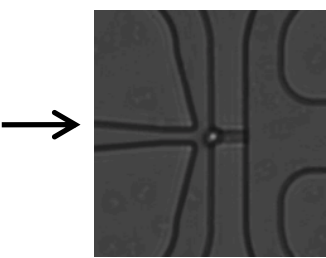

Captured SC

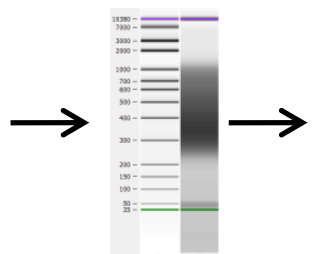

Library prep

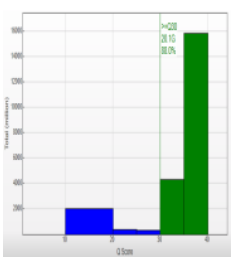

scRNA-Seq

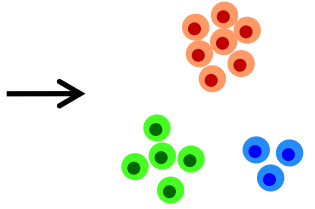

SC transcriptome

\section{B}

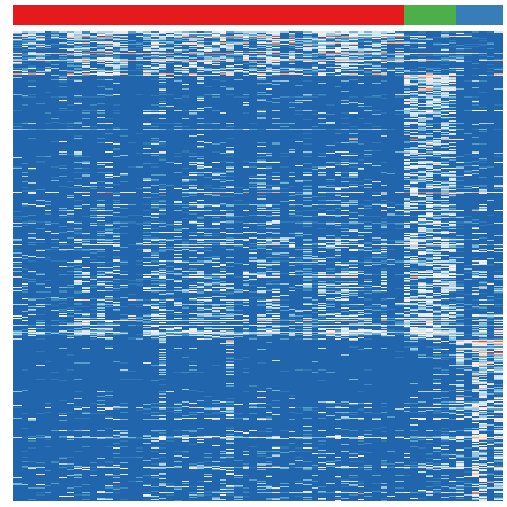

C

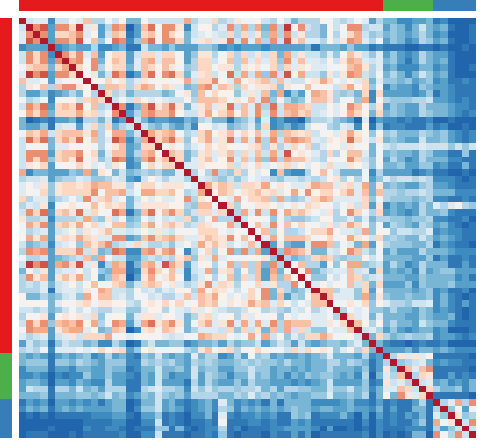

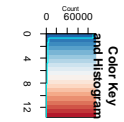

Cluster 1

Cluster 2

Cluster 3

D

kegg_pathways_in_cancer kegg_prostate_cancer kegg_melanoma

kegg adherens junction kegg_arrhythmogenic_right_ventricular_.. reactome_cell_cell_communication reactome_cell_junction_organization pid_ajdiss_2pathway kegg_endometrial_cancer reactome_cell_cell_junction_organization
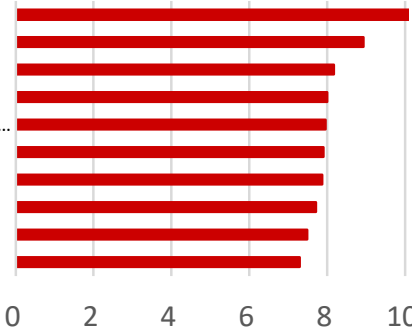

naba matrisome naba_core_matrisome naba_ecm_glycoproteins pid_integrin1_pathway

kegg_focal_adhesion naba_matrisome_associated pid_avb3_integrin_pathway reactome_extracellular_matrix_organizati.. reactome_developmental_biology reactome_axon_guidance
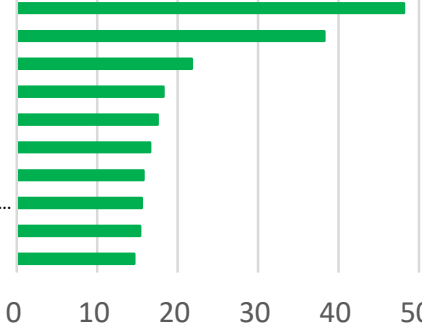
Cluster 3
Cluster 1 Cluster 2

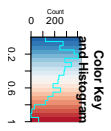

reactome_immune_system reactome_adaptive_immune_system reactome_hemostasis

kegg_chemokine_signaling_pathway reactome_platelet_activation_signaling_a... kegg_leishmania_infection

reactome_innate_immune_system reactome_cytokine_signaling in immune... reactome_g_alpha_i_signalling_events reactome_class_a1_rhodopsin_like_recep...

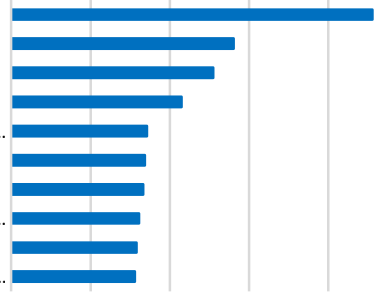




\section{A Fig 4}

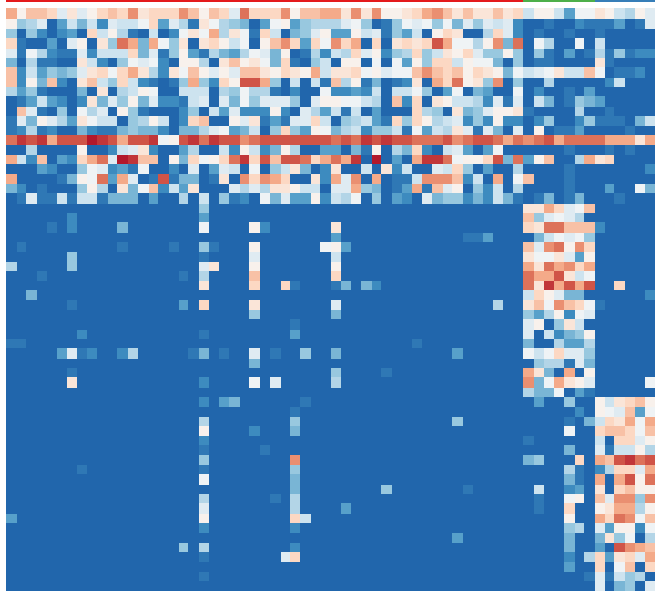

- Cluster 1

- Cluster_2

- Cluster_3

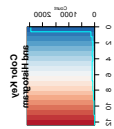

B

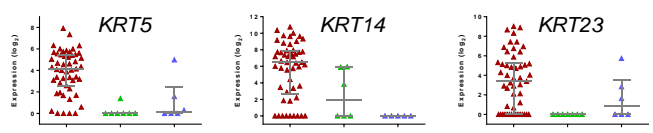

Cluster 1

Cluster_2

Cluster_3

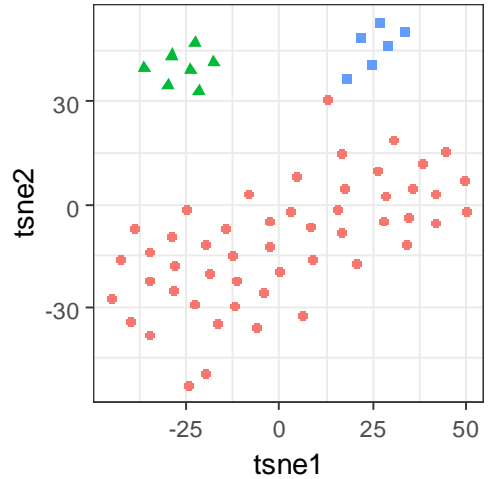

D

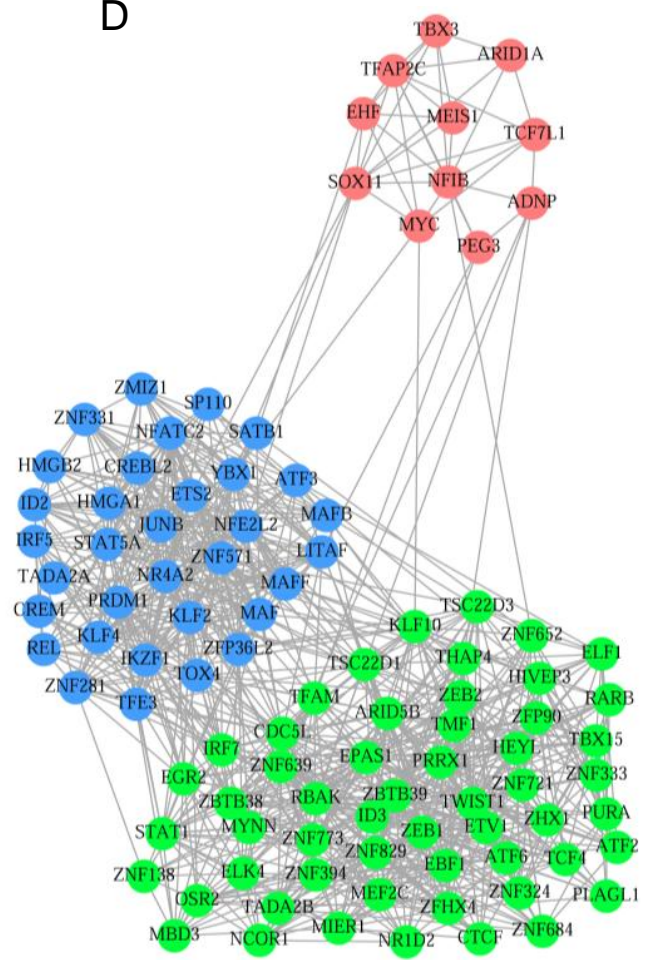




\section{A Fig 5}

B

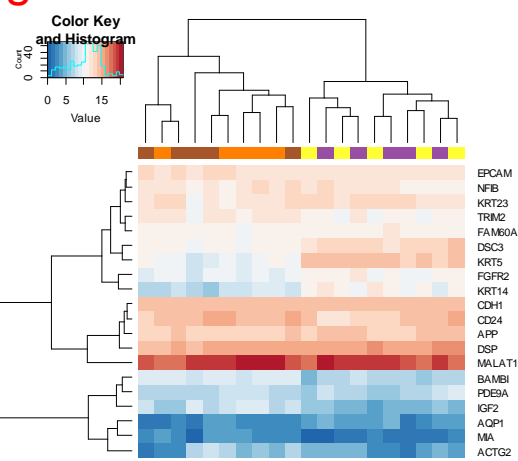

Benign $\mathrm{CD} 49 \mathrm{H} \mathrm{Hi}$

Benign CD49f Lo

Cancer CD49f
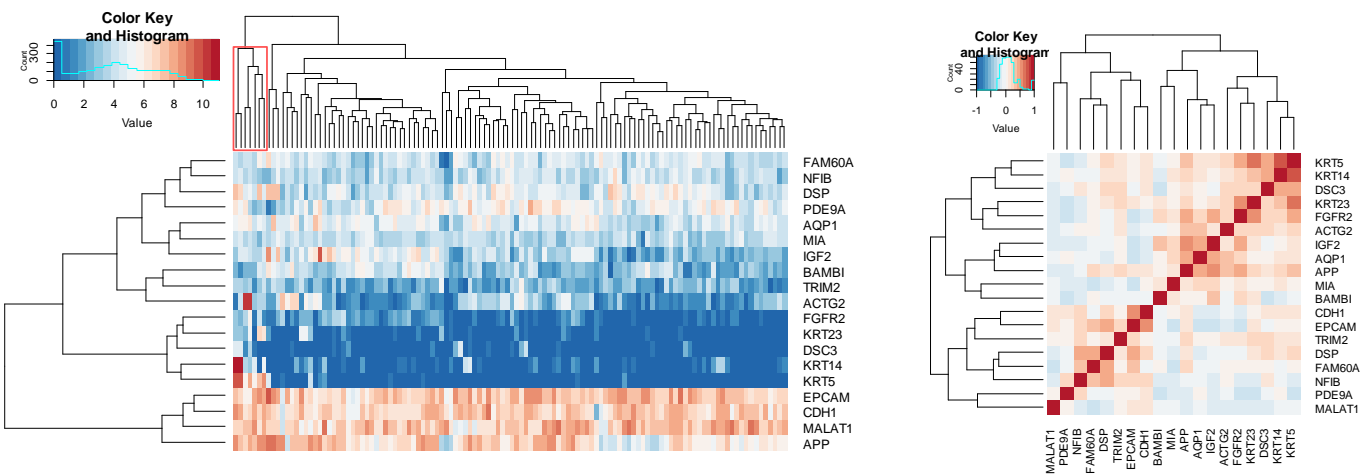

C
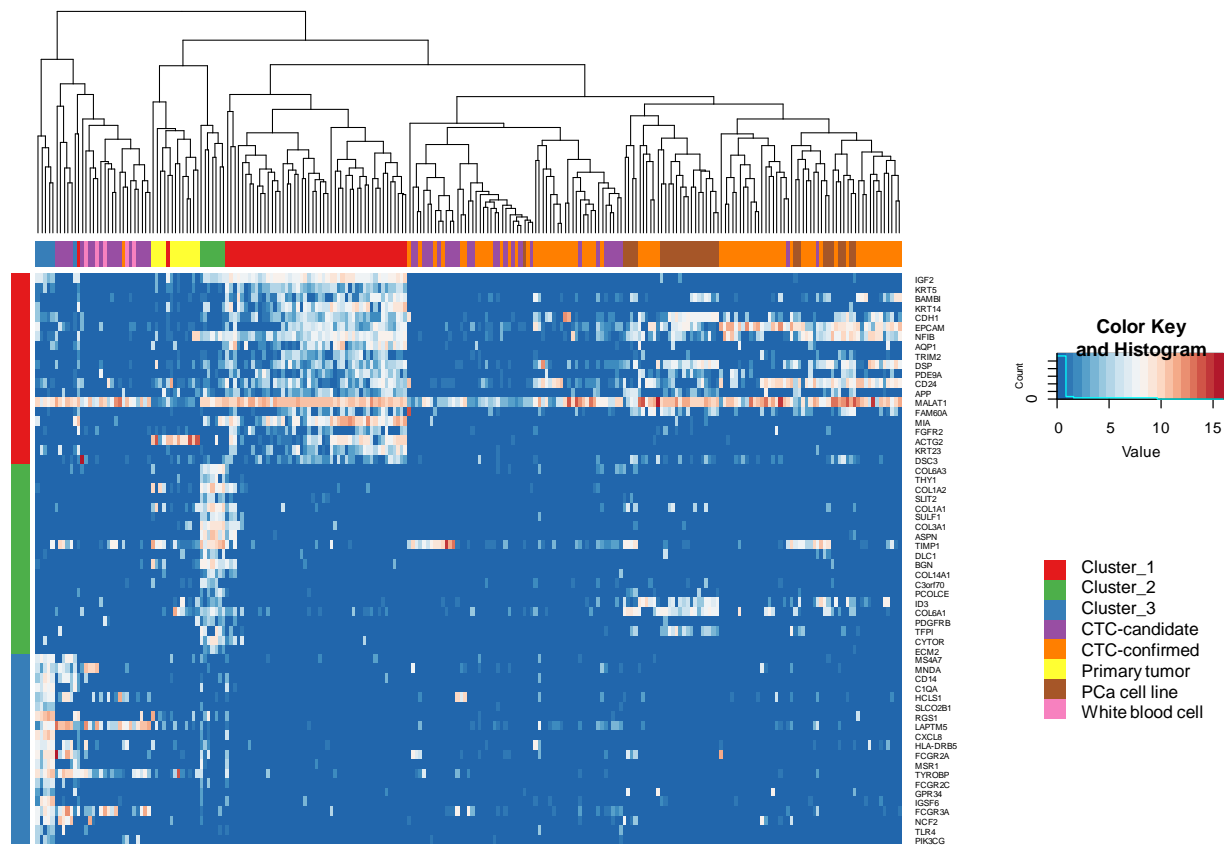